\title{
Early emission of rising optical afterglows: the case of GRB 060904B and GRB 070420^
}

\author{
A. Klotz ${ }^{1,2}$, B. Gendre ${ }^{3,4}$, G. Stratta ${ }^{5}$, A. Galli ${ }^{3,6}$, A. Corsi ${ }^{3}$, B. Preger ${ }^{5}$, S. Cutini ${ }^{5,7}$, A. Pélangeon ${ }^{8}$, J. L. Atteia ${ }^{8}$, \\ M. Boër ${ }^{1}$, and L. Piro \\ 1 Observatoire de Haute-Provence, 04870 Saint Michel l'Observatoire, France \\ 2 CESR, Observatoire Midi-Pyrénées, CNRS, Université de Toulouse, BP 4346, 31028 Toulouse Cedex 04, France \\ e-mail: klotz@cesr.fr \\ 3 Istituto di Astrofisica Spaziale e Fisica Cosmica, Sede di Roma, INAF, via Fosso del Cavaliere 100, 00133 Roma, Italy \\ 4 University degli Studi di Milano-Bicocca, Piazza dell'Ateneo Nuovo, 20126, Milano, Italy \\ ASI Science Data Center (ASDC), via G. Galilei, 00044 Frascati, Italy ${ }^{\star \star}$ \\ INFN-Trieste, Padriciano 99, 34012 Trieste, Italy \\ Università di Perugia, Dipartimento di fisica, viale A. Pascoli, 06123 Perugia, Italy \\ ${ }^{8}$ LATT, Observatoire Midi-Pyrénées, CNRS, Université de Toulouse, 14 avenue E. Belin, 31400 Toulouse, France
}

Received 14 September 2007 / Accepted 8 February 2008

\section{ABSTRACT}

\begin{abstract}
Aims. We present the time-resolved optical emission of gamma-ray bursts GRB 060904B and GRB 070420 during their prompt and early afterglow phases.

Methods. We used time resolved photometry from optical data taken by the TAROT telescope and time resolved spectroscopy at high energies from the Swift spacecraft instrument.

Results. The optical emissions of both GRBs are found to increase from the end of the prompt phase, passing to a maximum of brightness at $t_{\text {peak }}=9.2 \mathrm{~min}$ and $3.3 \mathrm{~min}$ for GRB 060904B and GRB 070420 respectively and then decrease. GRB 060904B presents a large optical plateau and a very large X-ray flare. We argue that the very large X-flare occurring near $t_{\text {peak }}$ is produced by an extended internal engine activity and is only a coincidence with the optical emission. GRB 070420 observations would support this idea because there was no X-flare during the optical peak. The nature of the optical plateau of GRB 060904B is less clear and might be related to the late energy injection.
\end{abstract}

Key words. gamma ray : bursts

\section{Introduction}

Since the discovery of Gamma-Ray Burst (GRB) X-ray afterglows, in 1997 (Costa et al. 1997), it has been possible to obtain a precise burst localization that allows the follow-up of GRBs from radio to X-ray (Wijers \& Galama 1999). From that date, tens of GRB optical afterglows have been detected by ground-based rapid response telescopes. The launch of the Swift satellite, in late 2004 (Gehrels et al. 2004) has increased the usefulness of robotic telescopes. In fact, the capability of Swift to alert other observatories within seconds after the burst, and then re-point quickly on the burst position has allowed us to significantly increase the number of successful afterglow optical detections and to perform early optical observations. The large amount of data collected during these years has shown that during the first phases (a few hundreds of seconds) the optical emission can be as bright as $m_{V} \sim 14-17$ before decreasing as $F(t) \propto t^{-\alpha} v^{-\beta}$, with $\alpha \sim 1.1-1.7$ (Fox 2005; Berger et al. 2005; De Pasquale et al. 2006; Gendre et al. 2006). Several

\footnotetext{
* Based on observations performed with TAROT at the Calern/OCA and La Silla/ESO observatories, GCN data archive and Swift public data archive.
}

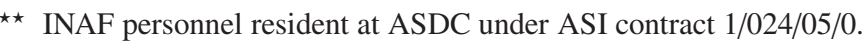

events were observed optically during the prompt phase ${ }^{1}$, e.g. GRB 990123 (Akerlof et al. 1999), GRB 041219A (Vestrand et al. 2006), GRB 050401 (Rykoff et al. 2005), GRB 050904 (Boër et al. 2006) or GRB 060111B (Klotz et al. 2006b). In some cases the optical and $\gamma$-ray prompt emissions appeared correlated (e.g. GRB 041219A) and are likely produced by internal shocks generated by variations in the energy ejection due to the central engine (Vestrand et al. 2006). In other cases (e.g. GRB 990123) the optical prompt emission is highly variable and not correlated with the $\gamma$-ray one; they can be produced by different source regions (i.e. internal and reverse shocks respectively), and/or by different emission mechanisms (Inverse Compton and synchrotron) (Akerlof et al. 1999).

Early optical afterglow data play a role in obtaining information on the physics of the central engine, and possibly in constraining the initial Lorentz factor of the fireball (e.g. Zhang et al. 2003). However, the beginning of the optical afterglow emission is often missed either because it occurs before the first observations are completed, or because it is hidden by the high brightness of an optical flash that sometimes occurs when the GRB is still active (e.g GRB 060111B in Klotz et al. 2006b). Sometimes, the optical afterglow takes a few minutes to reach its maximum and rapid response optical telescopes are able to

\footnotetext{
1 We call "prompt phase" the period during which Swift-BAT detected the high energy emission defined by the $T_{90}$ duration.
} 
record the rise of the light curve. For example this is the case of GRB 060418 and GRB 060607A (Molinari et al. 2007), which allowed investigators to constrain the initial fireball Lorentz factor to $\Gamma_{0} \sim 400$.

In this paper we report the early optical observations from $23 \mathrm{~s}$ to $43 \mathrm{~min}$ of GRB $060904 \mathrm{~B}$ and from $40 \mathrm{~s}$ to $18 \mathrm{~min}$ of GRB 070420 performed with the TAROT robotic observatories. These two GRBs have an optical afterglow that reaches a maximum hundreds of seconds after the GRB. We compare early simultaneous optical, X-ray and $\gamma$-ray data to address the question of the relation between optical and high energy emissions in the first minute after the GRB.

\subsection{GRB060904B}

GRB 060904B was detected on September 4th, 2006 at 02:31:03 UT by the BAT instrument on the Swift spacecraft (trigger $=228006$, Grupe et al. 2006). This GRB is a doublepeak event (Markwardt et al. 2006): the BAT light curve shows an initial fast rise exponential decay pulse $9 \mathrm{~s}$ wide at $2 \mathrm{~s}$ before the BAT trigger (hereafter $t_{\text {trig }}$ ) followed by a second, weaker peak detected within the $15-25 \mathrm{keV}$ range, starting at $t_{\text {trig }}+120 \mathrm{~s}$, peaking at $t_{\text {trig }}+155 \mathrm{~s}$ and finishing at $t_{\text {trig }}+220 \mathrm{~s}$. $T_{90}(15-350 \mathrm{keV})$ is $192 \pm 5 \mathrm{~s}$. Swift-XRT observations began at $t_{\text {trig }}+69 \mathrm{~s}$. The X-ray light curve displays a shallow emission during the gamma emission, followed by a huge flare. Optical ground follow-up began very early when the gamma emission was still active: ROTSE-IIIc (Rykoff et al. 2006) detected a source at RA $=03^{\mathrm{h}} 52^{\mathrm{m}} 50.52^{\mathrm{s}}$ Dec $=-00^{\circ} 43^{\prime} 30.85^{\prime \prime}(\mathrm{J} 2000,0)$ with $R=17.3$ at $t_{\text {trig }}+18.5 \mathrm{~s}$. Klotz et al. (2006a) reported a brightening of the optical transient reaching a peak of brightness $R \sim 17$ near $t_{\text {trig }}+400 \mathrm{~s}$. Optical spectroscopy was performed with VLT + FORS1 (Fugazza et al. 2006), at $t_{\text {trig }}+5.15 \mathrm{~h}$ observing several metallic absorption lines at $z=0.703$. Grupe et al. (2006) mentioned that Swift-UVOT detected the afterglow in a finding chart exposure of $246 \mathrm{~s}$ with the $V$ filter started $70 \mathrm{~s}$ after the BAT trigger. According to Schlegel et al. (1998) galactic extinction is $E(B-V)=0.172$ thus implying (assuming $R_{V}=3.1$ ) $A_{B}=0.74, A_{V}=0.57$ and $A_{R}=0.46$.

\subsection{GRB070420}

GRB 070420 was detected on April 20th, 2007 at 06:18:13 UT by the Swift-BAT instrument (trigger $=276321$, Stamatikos et al. 2007a). Swift instrument analysis was reported by Stamatikos et al. (2007b) providing celestial coordinates from UVOT at $\mathrm{RA}=08^{\mathrm{h}} 04^{\mathrm{m}} 55.17^{\mathrm{s}}$ Dec $=-45^{\circ} 33^{\prime} 20^{\prime \prime}(\mathrm{J} 2000,0)$. The BAT light curve shows a slow rise that began $50 \mathrm{~s}$ before the trigger. $T_{90}(15-350 \mathrm{keV})$ is $77 \pm 4 \mathrm{~s}$. Swift-XRT observations began at $t_{\text {trig }}+99 \mathrm{~s}$. The X-ray light curve displays a steep decay between $t_{\text {trig }}+106$ and $t_{\text {trig }}+300 \mathrm{~s}$. Then, a plateau occurred until at least $t_{\text {trig }}+2000 \mathrm{~s}$ before a decay until at least few $10^{4} \mathrm{~s}$. No X-flare was detected. D'Avanzo et al. (2007) observed the transient at $H=13.1 \pm 0.2$ with the REM telescope. From high energy spectral properties delivered by the KONUS experiment, Pélangeon $\&$ Atteia (2007) computed a pseudo-redshift $p z=1.56 \pm 0.35$. UVOT detection in the $U$ band (Immler et al. 2007) confirms the low redshift of the burst. According to Schlegel et al. (1998) galactic extinction is $E(B-V)=0.50$. Assuming $R_{V}=3.1$, the extinctions are thus $A_{B}=2.18, A_{V}=1.63, A_{R}=1.33$, $A_{H}=0.29$.
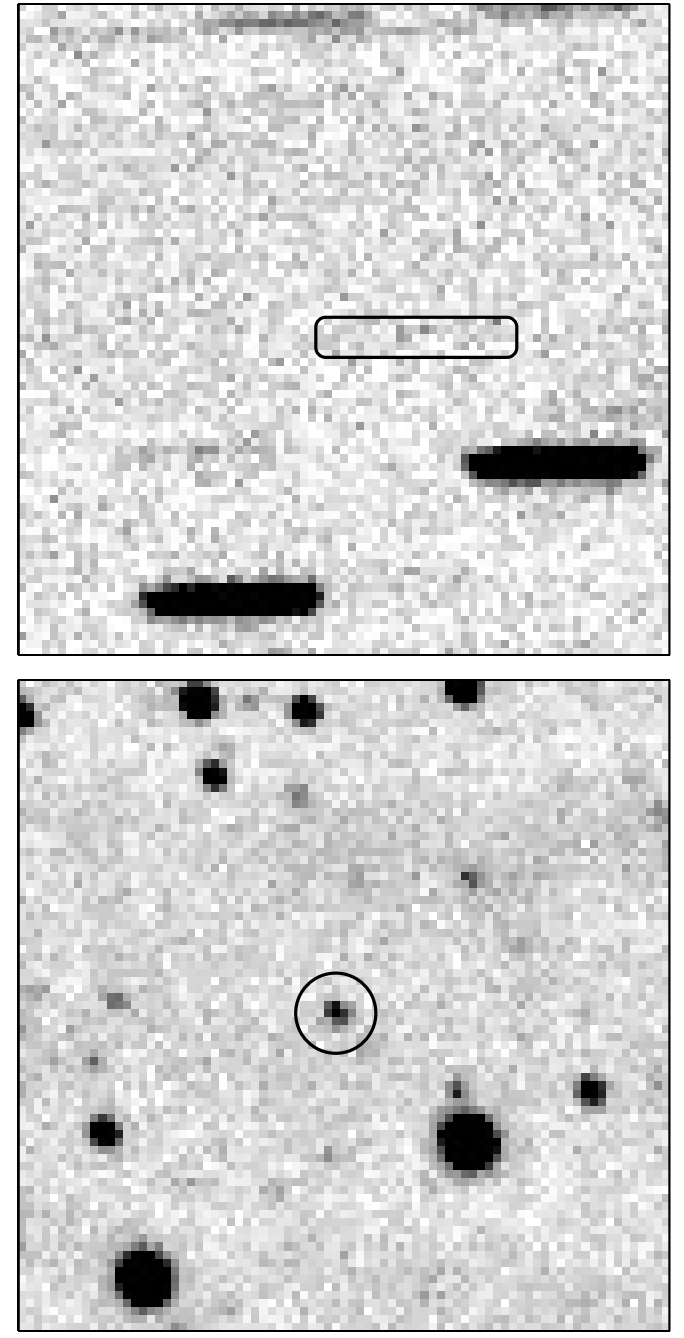

Fig. 1. GRB 060904B. Top: this image was taken between $23 \mathrm{~s}$ and $83 \mathrm{~s}$ after the GRB trigger. The hour angle velocity was adapted to obtain stars as trails of $\sim 20$ pixel length during the $60 \mathrm{~s}$ exposure. The theoretical position of the GRB trail is indicated by the rectangle. A cluster of 4 pixels is present near the center of the rectangle but is considered as noisy pixels or "cosmic" trace rather than an optical flash (see text). Bottom: sum of images taken by TAROT in diurnal motion mode. The optical transient position is indicated by the circle. The image size is 7 arcmin, North is up, East left.

\section{TAROT observations}

TAROT are two fully autonomous $25 \mathrm{~cm}$ aperture telescopes installed at Calern observatory (Observatoire de la Côte d'Azur - France) and at La Silla observatory (European Southern Observatory - Chile). These telescopes are devoted to very early observations of GRB optical counterparts. A technical description can be found in Bringer et al. (2001). GRB 060904B was observed with TAROT Calern and GRB 070420 with TAROT La Silla.

When the notice is received, the first image is an unfiltered $60 \mathrm{~s}$ exposure taken in drift mode (see Figs. 1 and 2 top, described in Klotz et al. 2006b). For each GRB, the image began during the prompt phase with a drift velocity of $3 \mathrm{~s} /$ pixel. Successive images were tracked on the diurnal motion. A first series of five unfiltered (hereafter filter $C$ ) $30 \mathrm{~s}$ images was followed by four series of three $90 \mathrm{~s}$ images filtered $C-C-R$ respectively. Finally, series of three $180 \mathrm{~s}$ images were taken with the 

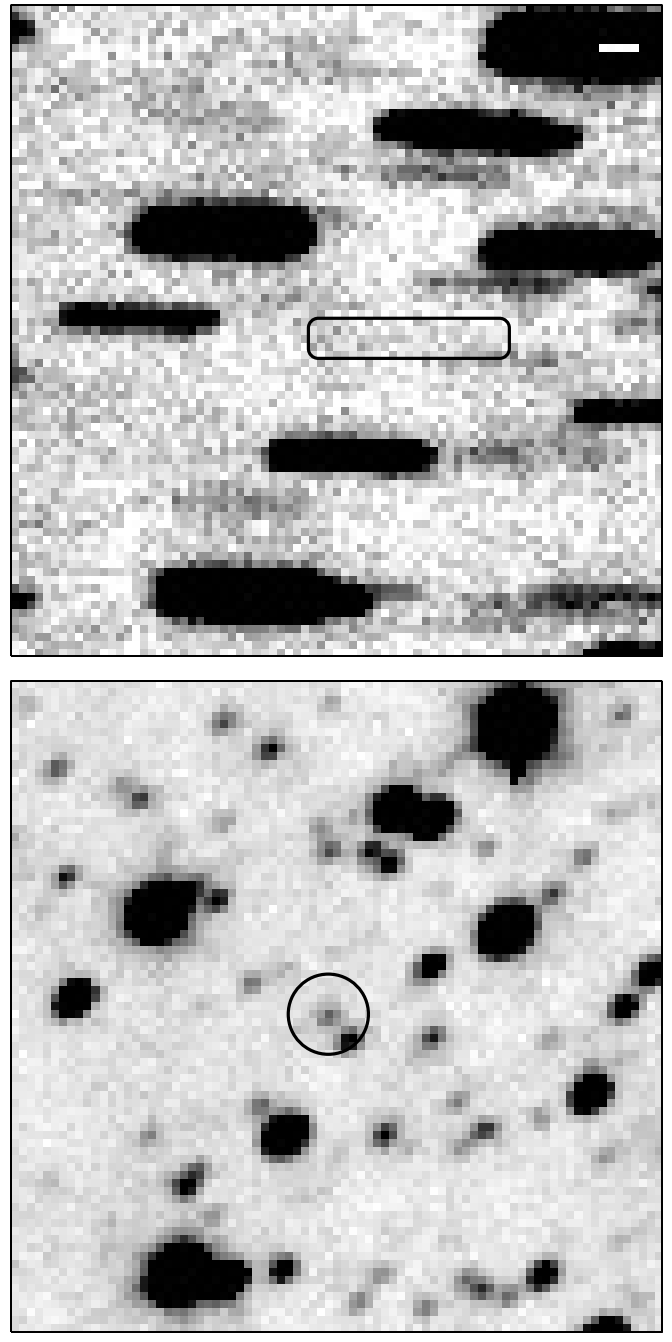

Fig. 2. GRB 070420. Top: this image was taken between $34 \mathrm{~s}$ and $94 \mathrm{~s}$ after the GRB trigger in drift mode (see Fig. 1). Three nearby stars (see Sect. 2.1) have been subtracted. The position of the GRB trail is indicated by the rectangle. A group of faint lightened pixels is present in the first third of the rectangle but we cannot conclude on its significance. Bottom: Sum of images taken by TAROT in diurnal motion mode. The optical transient position is indicated by the circle (nearby stars are not subtracted). The image size is 7 arcmin, North is up, East left.

same filter alternance. In the GRB 060904B case, the drift image began at $t_{\text {trig }}+23 \mathrm{~s}$ and entirely spans the prompt phase (that extends until $220 \mathrm{~s}$ after the trigger). Dawn arrived $43 \mathrm{~min}$ after the trigger and observations were stopped. For GRB 070420, the drift image began at $t_{\text {trig }}+34 \mathrm{~s}$. The field elevation decreased from 8 degrees above horizon to 5 degrees at the end of observations. Only the first 18 minutes of the event were recorded. Logs of observations are included in Tables 1 and 2.

\subsection{Image processing}

Photometry was performed using Point Spread Function (PSF) fitting taking a nearby star image as the PSF: USNO-B1 08920038669 for GRB 060904B and USNO-B1 0444-0107368 for GRB 070420. $C$ magnitudes were rescaled in the $R$ band considering the absence of dramatic spectral changes in the optical range. In the case of GRB 070420, three stars lie very close to the GRB location: USNO-B1 0444-0107222 (5", $R=17.3$ ), USNOB1 0444-0107218 (7", $R=$ 17.6), USNO-B1 0444-0107203 $\left(13^{\prime \prime}, R=15.6\right)$. We subtracted these stars using the PSF of USNO-B1 0444-0107368 $(R=13.3)$ before the PSF fitting of the GRB. Tables 1 and 2 give photometric results of both GRBs from TAROT and other useful measurements published in GCN Circulars.

\subsection{Trailed images}

At the GRB 060904B position (Fig. 1, top), no trail is detected at $R<17.2$ except for a small cluster of 4 pixels near $t_{\text {trig }}+42.8 \mathrm{~s}$. We first considered it as a possible optical flash associated with the GRB (Klotz et al. 2006a). The punctual shape implies that its duration is less than $3 \mathrm{~s}$ (the time sampling of the trail) corresponding to $R<15.9$. Rykoff (private communication) provided us with a series of $5 \mathrm{~s}$ exposures obtained with ROTSE-III telescopes. One of these images was taken between $t_{\text {trig }}+42.6 \mathrm{~s}$ and $t_{\text {trig }}+47.6 \mathrm{~s}$. It shows no object brighter than $R=16.7$. As a consequence, we do not confirm this flash to be real. From a statistical point of view, the signal to noise ratio $(\mathrm{S} / \mathrm{N})$ of the four involved pixels are : 1.0, 2.0, 2.7, 2.8. Considering a Gaussian noise distribution of the sky background, simultaneous values higher than the corresponding $\mathrm{S} / \mathrm{N}$ is $\sim 10^{-6}$ for four contiguous pixels. This excludes a natural background fluctuation. Thus, it is probably an artifact (e.g. a cosmic trace). We took this into account when we computed limiting magnitudes (see Fig. 3). From TAROT and ROTSE-III data, we conclude that the optical emission was $R>17.2$ during the prompt emission, except during $t_{\text {trig }}+41.3 \mathrm{~s}$ to $t_{\text {trig }}+44.3 \mathrm{~s}$ where it was $R>15.9$.

In the case of GRB 070420 (Fig. 2 top), a very faint trace is present at the beginning of the theoretical place of the trail but the signal to noise ratio is lower than 3 , preventing any conclusion.

\subsection{The optical afterglow of GRB 060904B}

The optical light-curve of GRB 060904B is shown in Fig. 3. Before the end of the prompt phase, the optical emission rose with a slope $\alpha_{1}=-0.82$ between $t_{\text {trig }}+90 \mathrm{~s}$ and $t_{\text {trig }}+550 \mathrm{~s}$ reaching $R=16.8$ at $t_{\text {trig }}+550 \mathrm{~s}$. Then, the flux decreased with a slope $\alpha_{2}=1.0$ down to $R=17.8$ at $t_{\text {trig }}+1270 \mathrm{~s}$. From this date, the flux remained at a constant level until the end of the available early observations $\left(t_{\text {trig }}+49.8 \mathrm{~min}\right)$. Late observations obtained 15 hs after the trigger imply a late decay index of $\alpha_{3}=1.03$ in the range $t_{\text {trig }}+$ [50 min-1.9 day].

Interpolating $R, B$ and $V$ magnitude measurements, we deduced $(B-R)$ at several dates (Table 3$)$. At $z=0.703$ (redshift of GRB 060904B) the color indexes are not affected by the Lyman- $\alpha$ cut-off (Roming et al. 1999). According to Simon et al. (1998), $(B-R)_{0}=0.8 \pm 0.3$ for typical early afterglows. The observed $(B-R)$ values of GRB 060904B, corrected for the galactic extinction, are compatible with a weak local extinction. Cobb \& Bailyn (2007) obtained data in the JHK bands during the late afterglow phase. They confirm a weak extinction. From published optical and near infrared data obtained during the late afterglow phase, Kann et al. (2008) derived the intrinsic extinction $A_{V}=0.44 \pm 0.05$ using a Small Magellanic Cloud dust model.

\subsection{The optical afterglow of GRB 070420}

The optical light-curve of GRB 070420 is shown in Fig. 4. After the end of the prompt phase, optical emission had risen at a rate $\alpha_{1}=-1.26$. This rising rate is compatible with $V$ band data 
Table 1. Log of the optical measurements of GRB 060904B from TAROT and GCN Circulars. $T$ are seconds since $t_{\text {trig. }} C$ magnitudes of TAROT images were translated to the $R$ photometric band by an appropriate offset. Errors $\Delta \mathrm{mag}$ are $2 \sigma$ for TAROT data.

\begin{tabular}{|c|c|c|c|c|}
\hline$T_{\text {start }}$ & $T_{\text {end }}$ & mag. & $\Delta \mathrm{mag}$ & GCNC Ref. \\
\hline 18.5 & 23.5 & $R 17.30$ & 0.10 & 5504 \\
\hline 23.0 & 41.3 & $C>17.2$ & & TAROT \\
\hline 41.3 & 44.3 & $C>15.9$ & & TAROT \\
\hline 44.3 & 86.0 & $C>17.2$ & & TAROT \\
\hline 57.8 & 147.8 & $R 18.21$ & 0.02 & 5511 \\
\hline 90.8 & 120.8 & C 18.38 & 0.47 & TAROT \\
\hline 128.1 & 157.4 & C 17.76 & 0.32 & TAROT \\
\hline 164.1 & 194.7 & C 17.99 & 0.35 & TAROT \\
\hline 71.0 & 316.0 & $V 18.64$ & 0.30 & 5519 \\
\hline 200.7 & 230.7 & $C 17.61$ & 0.26 & TAROT \\
\hline 237.8 & 267.9 & $C 17.15$ & 0.20 & TAROT \\
\hline 177.8 & 358.8 & B 18.32 & 0.04 & 5511 \\
\hline 283.5 & 373.5 & $C 17.31$ & 0.13 & TAROT \\
\hline 380.1 & 470.1 & C 17.02 & 0.11 & TAROT \\
\hline 387.8 & 477.8 & $R 17.01$ & 0.02 & 5511 \\
\hline 502.0 & 527.0 & $R 16.90$ & 0.12 & 5541 \\
\hline 479.7 & 569.7 & $R 16.78$ & 0.09 & TAROT \\
\hline 526.2 & 526.2 & $R 16.75$ & 0.02 & 5524 \\
\hline 597.6 & 597.6 & $R 16.86$ & 0.03 & 5524 \\
\hline 507.8 & 687.8 & $B 17.51$ & 0.04 & 5511 \\
\hline 578.0 & 668.0 & C 16.94 & 0.11 & TAROT \\
\hline 664.2 & 664.2 & $R 16.99$ & 0.03 & 5524 \\
\hline 653.0 & 678.0 & $R 17.09$ & 0.16 & 5541 \\
\hline 674.7 & 765.3 & C 17.06 & 0.11 & TAROT \\
\hline 735.0 & 735.0 & $R 17.05$ & 0.04 & 5524 \\
\hline 718.8 & 808.8 & $R 17.11$ & 0.02 & 5511 \\
\hline 784.8 & 784.8 & $R 17.22$ & 0.05 & 5524 \\
\hline 777.0 & 803.0 & $R 17.24$ & 0.19 & 5541 \\
\hline 774.3 & 864.3 & $R 17.22$ & 0.14 & TAROT \\
\hline 874.8 & 874.8 & $R 17.34$ & 0.06 & 5524 \\
\hline 889.0 & 914.0 & $R 17.36$ & 0.17 & 5541 \\
\hline 881.1 & 971.1 & C 17.52 & 0.17 & TAROT \\
\hline 838.8 & 1018.8 & $B 18.56$ & 0.04 & 5511 \\
\hline 1014.0 & 1014.0 & $R 17.48$ & 0.09 & 5524 \\
\hline 1004.0 & 1029.0 & $R 17.44$ & 0.14 & 5541 \\
\hline 978.3 & 1067.7 & C 17.54 & 0.16 & TAROT \\
\hline 1083.0 & 1083.0 & $R 17.50$ & 0.09 & 5524 \\
\hline 1073.0 & 1098.0 & $R 17.58$ & 0.14 & 5541 \\
\hline 1048.8 & 1138.8 & $R 17.79$ & 0.02 & 5511 \\
\hline 1077.3 & 1167.3 & $R 17.34$ & 0.16 & TAROT \\
\hline 1153.8 & 1153.8 & $R 17.64$ & 0.10 & 5524 \\
\hline 1175.6 & 1265.6 & C 17.56 & 0.16 & TAROT \\
\hline 1168.8 & 1348.8 & B 18.81 & 0.04 & 5511 \\
\hline 1272.8 & 1362.3 & C 17.93 & 0.22 & TAROT \\
\hline 1371.3 & 1461.8 & $R 17.86$ & 0.24 & TAROT \\
\hline 1378.8 & 1468.8 & $R 18.21$ & 0.02 & 5511 \\
\hline 1466.0 & 1491.0 & $R 17.70$ & 0.22 & 5541 \\
\hline 1505.0 & 1530.0 & $R 17.75$ & 0.20 & 5541 \\
\hline 1479.3 & 1659.8 & C 17.63 & 0.17 & TAROT \\
\hline 1669.0 & 1694.0 & $R 17.94$ & 0.18 & 5541 \\
\hline 1665.8 & 1846.5 & C 17.96 & 0.20 & TAROT \\
\hline 1712.8 & 1802.8 & $R 17.66$ & 0.02 & 5511 \\
\hline 1751.0 & 1776.0 & $R 17.79$ & 0.21 & 5541 \\
\hline 1802.0 & 1827.0 & $R 17.89$ & 0.14 & 5541 \\
\hline 1854.8 & 2034.8 & $R 17.67$ & 0.17 & TAROT \\
\hline 2044.5 & 2223.8 & C 17.78 & 0.20 & TAROT \\
\hline 2231.1 & 2410.4 & C 17.96 & 0.18 & TAROT \\
\hline 2242.8 & 2422.8 & $R 17.78$ & 0.02 & 5511 \\
\hline 2419.5 & 2600.0 & $R 17.61$ & 0.22 & TAROT \\
\hline 2538.8 & 2718.8 & $R 17.79$ & 0.02 & 5511 \\
\hline 2805.8 & 2985.8 & $R 17.99$ & 0.02 & 5511 \\
\hline 56592.0 & 56592.0 & $R 21.40$ & 0.20 & 5548 \\
\hline 76529.4 & 77969.4 & $R 21.63$ & 0.18 & 5741 \\
\hline 86477.4 & 87377.4 & $R 21.75$ & 0.11 & 5526 \\
\hline 87514.2 & 88414.2 & $R 21.74$ & 0.12 & 5526 \\
\hline 89415.0 & 90315.0 & $R 21.80$ & 0.20 & 5526 \\
\hline 162531.0 & 163731.0 & $R 22.40$ & 0.30 & 5741 \\
\hline
\end{tabular}

Table 2. Log of the optical measurements of GRB 070420 from TAROT and GCN Circulars. Columns and units are the same as Table 1.

\begin{tabular}{ccccc}
\hline \hline$T_{\text {start }}$ & $T_{\text {end }}$ & mag. & $\Delta$ mag. & GCNC Ref. \\
\hline 39.9 & 48.9 & $C>16.75$ & & TAROT \\
48.9 & 57.3 & $C>16.45$ & & TAROT \\
57.3 & 65.7 & $C>16.25$ & & TAROT \\
65.7 & 74.7 & $C>16.75$ & & TAROT \\
74.7 & 83.1 & $C>16.75$ & & TAROT \\
83.1 & 91.5 & $C>16.75$ & & TAROT \\
90.0 & 100.0 & $V 17.90$ & 0.10 & 6336 \\
101.1 & 131.0 & $C 16.64$ & 0.18 & TAROT \\
137.6 & 166.4 & $C 16.46$ & 0.13 & TAROT \\
109.0 & 208.0 & $V 17.40$ & 0.10 & 6336 \\
173.1 & 203.1 & $C 16.03$ & 0.11 & TAROT \\
296.1 & 386.1 & $C 16.38$ & 0.12 & TAROT \\
392.1 & 482.0 & $C 16.73$ & 0.17 & TAROT \\
491.6 & 582.3 & $R 16.84$ & 0.23 & TAROT \\
590.0 & 680.0 & $C 17.05$ & 0.31 & TAROT \\
693.0 & 703.0 & $B 18.80$ & 0.10 & 6336 \\
686.0 & 776.0 & $C 17.05$ & 0.26 & TAROT \\
890.0 & 980.1 & $C 17.50$ & 0.41 & TAROT \\
986.1 & 1075.5 & $C 17.65$ & 0.47 & TAROT \\
10530.0 & 11154.0 & $R 19.70$ & 0.40 & 6334 \\
12678.0 & 13308.0 & $I 19.30$ & 0.50 & 6334 \\
\hline
\end{tabular}

Table 3. Color indexes for GRB 060904B. $T_{\text {mean }}$ is the time since $t_{\text {trig }}$ when the color indice is measured. $T_{\text {mean }}$ is expressed in seconds. Column "mag." is not corrected for galactic extinction. Column "dereddened" is the color corrected by the galactic extinction.

\begin{tabular}{|c|c|c|c|c|c|}
\hline$\overline{T_{\text {mean }}}$ & Color & mag. & $\overline{\text { Deredened }}$ & Uncert. & $\overline{\text { Remarks }}$ \\
\hline 193 & $V-R$ & 1.0 & 0.89 & 0.3 & bad sampling \\
\hline 268 & $B-R$ & 0.93 & 0.65 & 0.04 & optical rising \\
\hline 598 & $B-R$ & 0.72 & 0.44 & 0.04 & $\begin{array}{l}\text { close to the top } \\
\text { of the bump }\end{array}$ \\
\hline 929 & $B-R$ & 1.10 & 0.82 & 0.04 & optical decreasing \\
\hline 1259 & $B-R$ & 1.0 & 0.72 & 0.04 & optical shallowing \\
\hline 4425 & $B-R$ & 0.58 & 0.30 & 0.15 & late afterglow \\
\hline 4831 & $V-R$ & 0.64 & 0.53 & 0.36 & late afterglow \\
\hline 5649 & $B-R$ & 0.88 & 0.60 & 0.28 & late afterglow \\
\hline
\end{tabular}

provided by UVOT (Immler et al. 2007). The optical data show a temporal gap at the maximum of the emission (due to a technical problem). Extrapolating the data, we estimate the peak of emission to occur near $t_{\text {trig }}+3.3$ min with a magnitude of $R=15.9$. Then the flux decreased at a rate of $\alpha_{2}=0.89$ to $R=17.6$ at $t_{\text {trig }}+17.2 \mathrm{~min}$. Later observations obtained 3 hours after the trigger are compatible with this decay. If we set the $t_{\text {trig }}$ time to the onset of the start of the BAT emission $(50 \mathrm{~s}$ earlier than the trigger time) we obtain $\alpha_{1}=-1.69$ and $\alpha_{2}=+0.91$.

Interpolating $R$ and some other band magnitude measurements we deduced color indexes at several epochs (Table 4). At $t_{\text {trig }}+95 \mathrm{sdec},(V-R)=+0.5$ (corrected for galactic extinction). At $t_{\text {trig }}+698 \mathrm{~s}$, during the optical decreasing phase, $(B-R)=+0.85$.

\section{High energy observations}

\subsection{BAT analysis of GRB 060904B}

We have performed temporal and spectral analysis of the BAT data for GRB 060904B. Figure 5 shows the light curve in the 15-25 and 100-150 keV ranges: the numbered vertical columns 


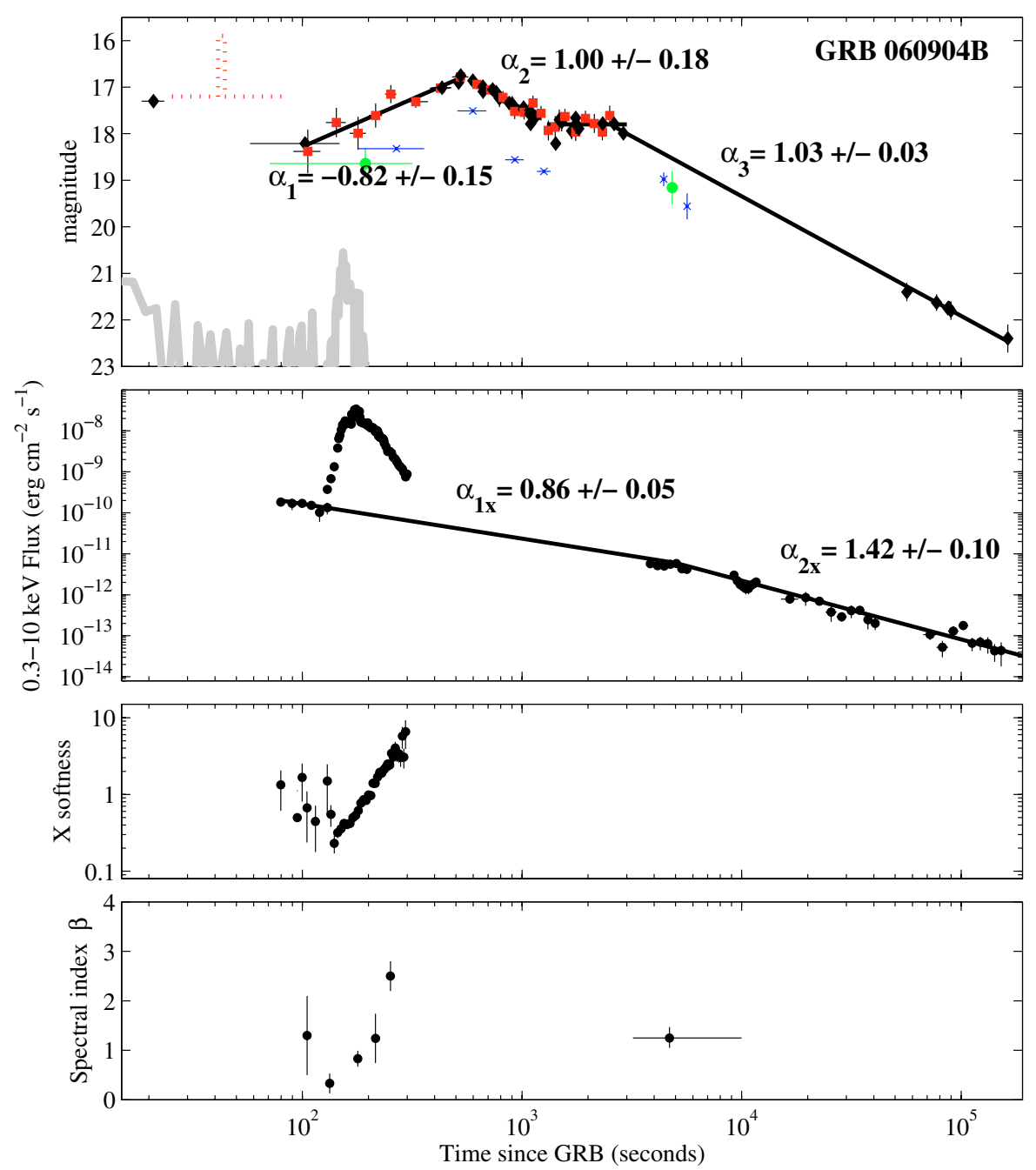

Fig. 3. Time resolved parameters of GRB 060904B. Top panel: optical lightcurve. Data are provided from Table 1. Red squares are TAROT data (plus the limiting magnitude during $23 \mathrm{~s}$ to $83 \mathrm{~s}$ indicated by the dotted line). Black diamonds are $R$ measurements of other observatories. Green disk and blue $\mathrm{x}$ are from the literature for the $V$ and $B$ band respectively. The BAT light curve is displayed as the light gray curve offset arbitrarily. Fits are based on the flux law: $f(t) \propto t^{-\alpha}$. Second panel: X-ray light-curve from XRT data. The count rate has been converted to flux units using the best fit spectral model of late $\mathrm{X}$-ray afterglow (to avoid spurious features due to the large spectral variations observed during the flare). Third panel: softness ratio defined by $(0.3-1.5 \mathrm{keV}) /(1.5-10 \mathrm{keV})$. Bottom panel: $\mathrm{X}$-ray band spectral index.
Table 4. Color indexes for GRB 070420. See column descriptions in Table 3.

\begin{tabular}{cccccc}
\hline \hline$T_{\text {mean }}$ & Color & mag. & Deredened & Uncert. & Remarks \\
\hline 95 & $V-R$ & 0.8 & 0.5 & 0.2 & optical rising \\
300 & $R-H$ & 3.15 & 2.1 & 0.1 & optical decreasing \\
698 & $B-R$ & 1.7 & 0.85 & 0.1 & optical decreasing \\
\hline
\end{tabular}

refer to the TAROT observation periods with a detected optical source. No high energy precursor is detectable up to $t_{\text {trip }}-200 \mathrm{~s}$. No signal was detected by BAT during the first TAROT observation (window number 1), whereas observations number 2 and 3 cover the second and much fainter peak in the high energy light curve. In order to obtain broad band time-resolved spectra for GRB 060904B, we performed spectral analysis of the BAT data in three different epochs: the main peak, visible only by BAT (from $t_{\text {trip }}-5 \mathrm{~s}$ to $t_{\text {trip }}+25 \mathrm{~s}$ ), the rise of the second peak (from $t_{\text {trig }}+123.9 \mathrm{~s}$ to $t_{\text {trig }}+159.9 \mathrm{~s}$ ), and the decay of the second peak (from $t_{\text {trig }}+159.9 \mathrm{~s}$ to $t_{\text {trig }}+197.1 \mathrm{~s}$ ). The second and third time intervals are slightly longer than (but centered on) the two corresponding TAROT observations $\left(t_{\text {trig }}+[128.1-157.4] \mathrm{s}\right.$ and $t_{\text {trig }}+$ [164.1-194.7] s respectively). This choice was made in order to increase the signal to noise ratio and thus the quality of the obtained spectra while maintaining a good simultaneity of the observations.
The 15-150 keV energy spectra during all the 2 and 3 TAROT epochs are well fitted by a simple power law model. We find clear evidence of spectral softening through the three epochs: the photon index is in fact $\Gamma_{1}=1.35 \pm 0.1$ during the main peak, $\Gamma_{2}=2.16 \pm 0.3$ during observation number 2 and $\Gamma_{3}=2.34 \pm 0.4$ during observation number 3 . The spectral softening is also confirmed by a comparison of the light curves in the $15-25 \mathrm{keV}$ and 100-150 keV energy bands (Fig. 5).

The fluence in the first peak is $1.22 \times 10^{-6} \mathrm{erg} \mathrm{cm}^{-2}$, in the second peak (observations 2 and 3 together) $3.68 \times 10^{-7} \mathrm{erg} \mathrm{cm}^{-2}$ for a total fluence of $1.6 \times 10^{-6} \mathrm{erg} \mathrm{cm}^{-2}$.

\subsection{XRT analysis of GRB $060904 B$}

The XRT began observing $69 \mathrm{~s}$ after the BAT trigger (Grupe et al. 2006). The data were processed using the ftools version 6.1.2. Data taken in window timing mode suffered from severe pileup during the flare and were corrected using the prescriptions indicated in Romano et al. (2006). We used a rectangle box extraction region excluding 2 central pixels for the time intervals between $t_{\text {trig }}+145 \mathrm{~s}$ and $t_{\text {trig }}+166 \mathrm{~s}$ and between $t_{\text {trig }}+182.9 \mathrm{~s}$ and $t_{\text {trig }}+237.3 \mathrm{~s}$, and 5 central pixels for the time interval between $t_{\text {trig }}+166.0 \mathrm{~s}$ and $t_{\text {trig }}+182.9 \mathrm{~s}$. The extraction region was 50 pixel large. We corrected for the effects induced by a bad column and the pile-up excluded region located within the extraction region by estimating from the Ancillary Response 


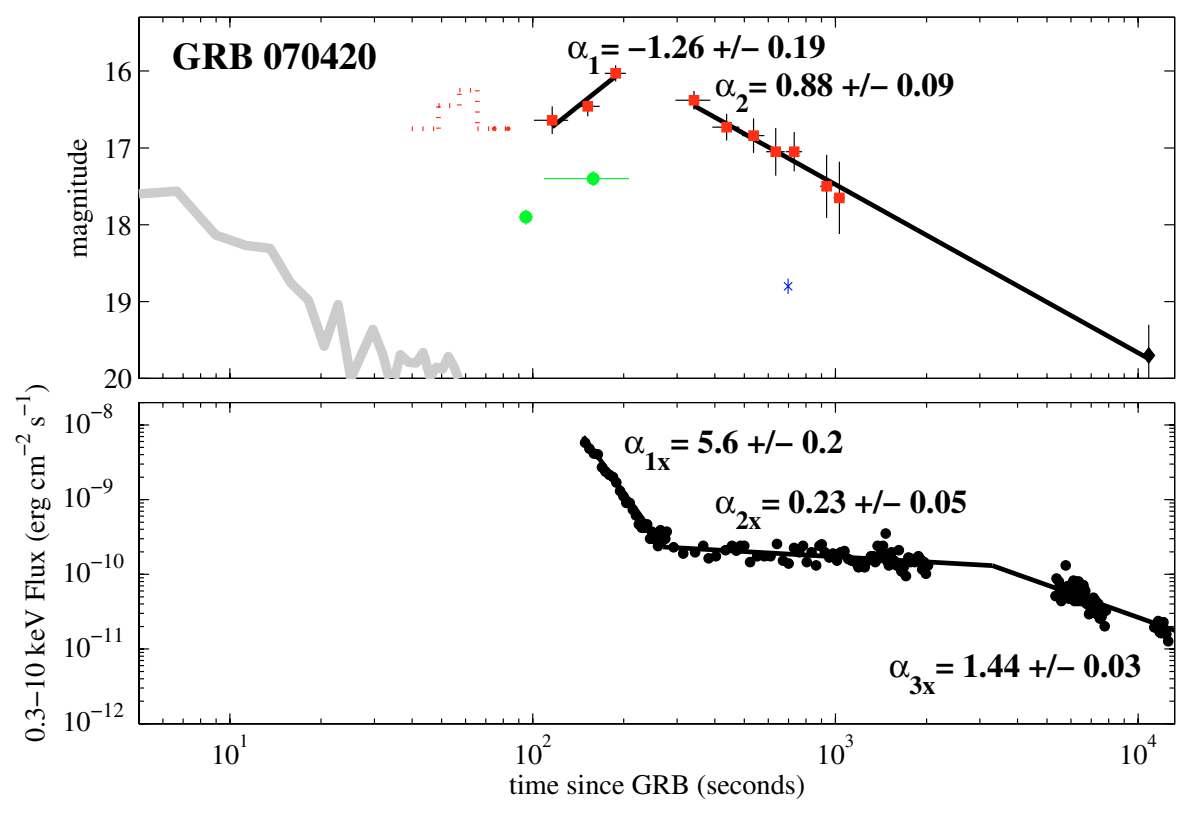

Fig. 4. Global light-curves of GRB 070420. Data are from Table 2. Red squares are TAROT data (plus the limiting magnitude during $40 \mathrm{~s}$ to $91 \mathrm{~s}$ indicated by the dotted line). Black diamonds are $R$ measurements of other observatories. The green disk and blue $\mathrm{x}$ are from the literature for the $V$ and $B$ band respectively. The BAT light curve is displayed as the light gray curve.

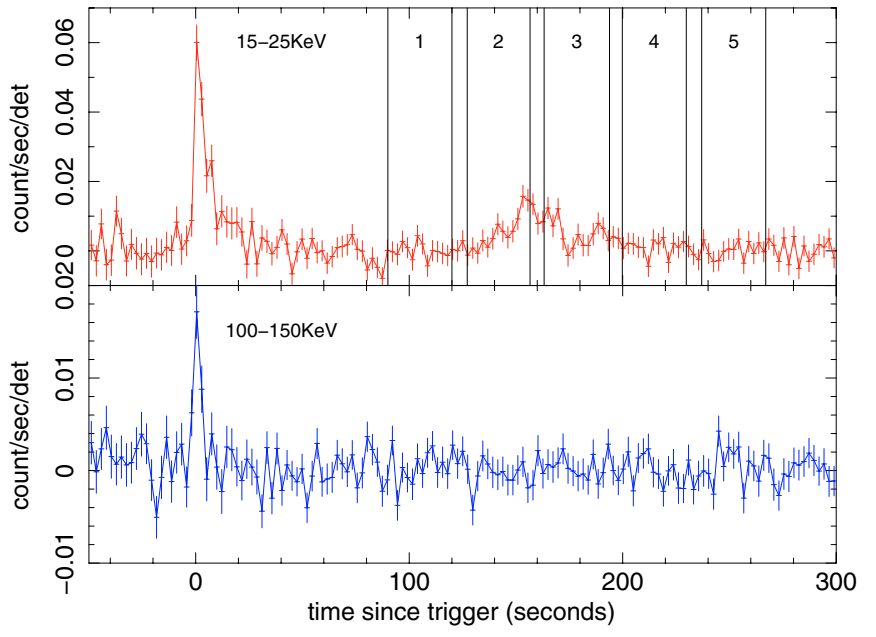

Fig. 5. BAT light curves at different energy ranges for GRB 060904B. The second peak near $t_{\text {trig }}+160 \mathrm{~s}$ is only visible in the softest energy band. The vertical lines define the 5 intervals coincident with the TAROT observations.

File the fraction of lost counts, and corrected all count rates for this loss. The light curve was extracted within the $0.3-10.0 \mathrm{keV}$ band and rebinned in order to obtain at least 25 counts per bin. All decay indexes indicated below are derived by fiting power laws using the $\chi^{2}$ statistic. As the $0.3-2.0 \mathrm{keV}$ range is not free from absorption, we prefered, for comparison with other works, to produce the figures using the standard $2.0-10.0 \mathrm{keV}$ band (free of absorption). We thus converted all $0.3-10.0 \mathrm{keV}$ count rates into a standard $2.0-10.0 \mathrm{keV}$ flux by using the appropriate mean conversion factor derived from the spectral analysis (see below). The light curve shows an initial shallow decay between $77.3 \mathrm{~s}$ and $\sim 138 \mathrm{~s}$ followed by a giant flare (temporal index rise $=-17.83 \pm 0.02$, temporal decay index $=6.1 \pm 0.4$ ) with a duration of $490 \mathrm{~s}$ (see Fig. 3). This flare has a $0.3-10.0 \mathrm{keV}$ mean flux of $2.2 \times 10^{-9} \mathrm{erg} \mathrm{s}^{-1} \mathrm{~cm}^{-2}$ and a total fluence of $8.8 \times 10^{-7} \mathrm{erg} \mathrm{cm}^{-2}$. The data feature a temporal gap between $\sim 300$ and $\sim 3500 \mathrm{~s}$. However, the data taken before and after the flare are consistent with a continuous and smooth power law decay with index $\alpha_{1 x}=0.86 \pm 0.05$ followed by a break near
$(5 \pm 1) \times 10^{3} \mathrm{~s}$ and a final decay with index $\alpha_{2 x}=1.42 \pm 0.1$. We do not find evidence of a plateau phase in X-ray.

We extracted the XRT spectra during each TAROT temporal bin (see Table 5), plus a global spectrum in order to derive a mean count-to-flux conversion factor for light curve conversions. All spectra were rebinned in order to include 20 net counts within each bin, and fit using the $\chi^{2}$ statistic. The spectral model was composed of a power law continuum absorbed by our galaxy (Galactic hydrogen column density fixed at $1.21 \times 10^{21} \mathrm{~cm}^{-2}$, Dickey \& Lockman 1990) and by the host galaxy at $z=0.703$. When possible, we fit the BAT and XRT spectra together. During the flare, the spectrum softens but more interestingly the foreground hydrogen column increases during the rising phase of the flare and then it decreases. This is a quite uncommon behavior and we interpret it as an artificial effect of a wrong assumption on the intrinsic spectral model. A Band model or cut-off power law provides a more realistic behavior (see Table 5), with a constant extragalactic absorption of $N_{H}=(8 \pm 2) \times 10^{21} \mathrm{H} \mathrm{cm}^{-2}$ (at $z=$ 0.703 ), and an hard-to-soft behavior, characteristic of prompt related emission. Very similar results have been obtained in the past for another giant X-ray flare associated with GRB 050502B (Falcone et al. 2006). The difference with GRB 060904B is that no rising optical afterglow was detected during the flare (or after). The late XRT data of GRB 060904B (from 3700 to $\sim 6000 \mathrm{~s}$ ) are compatible with a simple absorbed power law (extragalactic $N_{H}=(5 \pm 2) \times 10^{21} \mathrm{H} \mathrm{cm}^{-2}, \beta=1.3 \pm 0.3, \chi_{v}^{2}=1.21$, 19 d.o.f.). Compared to the spectral index found for the early XRT data (before the giant X-ray flare, see Table 5), no significant changes are observed within the errors.

\subsection{BAT analysis of GRB 070420}

We have performed temporal and spectral analysis of the BAT data for GRB 070420. The light curve, visible in Fig. 6 in the 15-350 keV energy range, shows a slow rise beginning about $50 \mathrm{~s}$ before the BAT trigger, then a multi-peaked structure and finally a gradual decay ending about $100 \mathrm{~s}$ after the trigger. Again, the vertical columns refer to the TAROT observing windows, but for this GRB no signal was detected by BAT that could be used to derive simultaneous broad band spectra at those epochs. 
Table 5. Spectral analysis of GRB 060904B. We indicate only the best fit models (top panel, a cut-off power law; bottom panel, a Band model). Column Bin refers to the TAROT measurements defined in Sect. 3.1 and reported in Fig. 5. Bins 1, 4, and 5 are XRT data only, while bins 2 and 3 are XRT and BAT data fit together. Values between parentheses are set to the value indicated. Extragalactic absorption is computed in the GRB rest frame. The two spectral indexes of the Band function are given as low energy spectral index first, high energy spectral index after. Several values are not constrained by the fit (the $E_{0}$ values for bins 1 and 5 with a cut-off power law). All errors are given at the $90 \%$ confidence level. When applicable, upper limits are given at the $95 \%$ confidence level.

\begin{tabular}{|c|c|c|c|c|c|c|}
\hline $\begin{array}{c}\text { Epoch } \\
\text { (seconds } \\
\text { since trigger) }\end{array}$ & Bin & $\begin{array}{l}\text { Spectral } \\
\text { index }\end{array}$ & $\begin{array}{c}E_{0} \\
(\mathrm{keV})\end{array}$ & $\begin{array}{l}\text { Extragalactic } \\
\text { absorption } \\
\left(10^{22} \mathrm{~cm}^{-2}\right)\end{array}$ & $\chi_{v}^{2}$ & d.o.f. \\
\hline $90.8-120.8$ & 1 & $1.3 \pm 0.8$ & - & $(0.78)$ & 0.92 & 1 \\
\hline $127.2-156.6$ & 2 & $0.3 \pm 0.2$ & $37.9_{-14.5}^{+36.6}$ & $0.8 \pm 0.5$ & 1.08 & 28 \\
\hline $163.2-193.8$ & 3 & $0.8 \pm 0.2$ & $13.2_{-4.1}^{+7.05}$ & $0.8 \pm 0.2$ & 1.18 & 119 \\
\hline $199.8-229.8$ & 4 & $1.2 \pm 0.5$ & $6.8_{-3.8}^{+13.4}$ & $0.6 \pm 0.2$ & 0.93 & 61 \\
\hline $237.0-267.0$ & 5 & $2.5 \pm 0.3$ & - & $0.8 \pm 0.2$ & 1.09 & 42 \\
\hline $127.2-156.6$ & & $\begin{array}{c}0.3 \pm 0.2 \\
>6.84\end{array}$ & $39.6_{-15.4}^{+35.7}$ & $0.8 \pm 0.5$ & 1.12 & 27 \\
\hline $163.2-193.8$ & & $\begin{array}{l}0.6 \pm 0.4 \\
1.8 \pm 0.3\end{array}$ & $5.7_{-2.9}^{+4.2}$ & $0.7 \pm 0.2$ & 1.05 & 118 \\
\hline $199.8-229.8$ & & $\begin{array}{c}1.3 \pm 0.5 \\
-\end{array}$ & $7.4_{-3.9}^{+4.1}$ & $0.6 \pm 0.1$ & 0.95 & 60 \\
\hline $237.0-267.0$ & & $\begin{array}{c}- \\
2.4 \pm 0.2\end{array}$ & $<3.59$ & $0.7 \pm 0.2$ & 1.11 & 41 \\
\hline
\end{tabular}

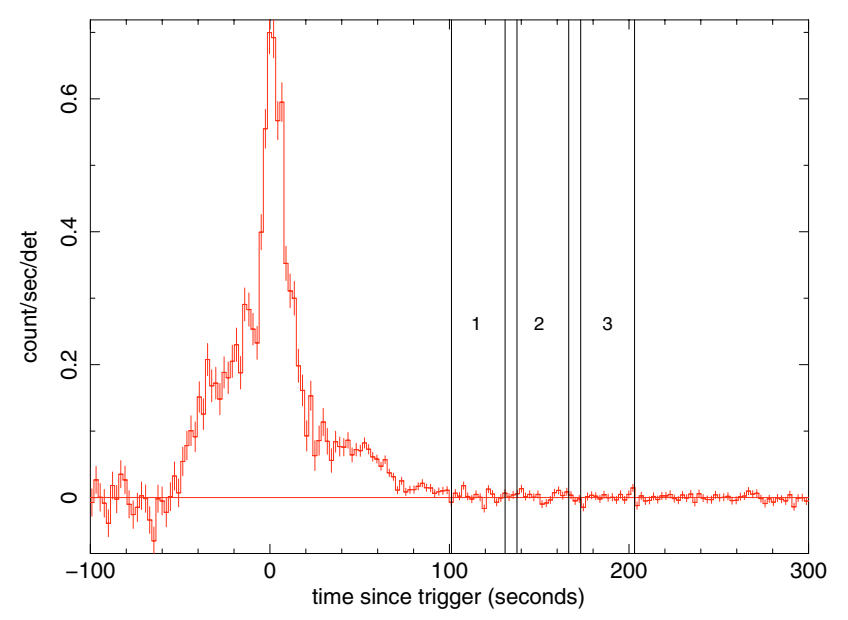

Fig. 6. BAT total light curve (15-350 keV) for GRB 070420. The vertical lines define the 3 intervals coincident with the TAROT observations. Note that a signal due to GRB 070420 is detected up to $\sim 50 \mathrm{~s}$ before the trigger time.

We have therefore performed the spectral analysis using the signal integrated over the BAT $T_{90}$ : a simple power law fits the observed spectrum between 15 and $150 \mathrm{keV}$ with $\Gamma=1.54 \pm$ 0.06 , while the burst fluence is $1.43 \times 10^{-5} \mathrm{erg} \mathrm{cm}^{-2}$.

We observed no significant spectral evolution during the emission detected by BAT.

\subsection{XRT analysis of GRB 070420}

The XRT observation starts $99.0 \mathrm{~s}$ after the BAT trigger; and $159 \mathrm{~s}$ after the start of the gamma-ray emission (which we define as the start of the event). The $0.3-10.0 \mathrm{keV}$ light curve is very smooth and features the standard "steep-flat-steep" behavior observed in the Swift era (O'Brien et al. 2005), with no flare superimposed (see Fig. 4). The three segments decay as $\alpha_{1 x}=$ $5.6 \pm 0.2, \alpha_{2 x}=0.23 \pm 0.05$ and $\alpha_{3 x}=1.44 \pm 0.03$ respectively, with two break times of $t_{b 1}=(3 \pm 1) \times 10^{2}$ and $t_{b 2}=$ $(3.3 \pm 0.2) \times 10^{3} \mathrm{~s}$. Note that these values differ from the ones reported in Stratta et al. (2007), as the start time of the event is different (Stratta et al. 2007, used the trigger time as $T_{0}$, while we used the start of the event as $T_{0}$ ).

The PC data suffer from a moderate pile-up during 280.2 and $\sim 7800 \mathrm{~s}$ after the trigger. The initial window timing data provide a good fit with a simple absorbed power law, and are consistent with no spectral variations (spectral index $\beta=1.5 \pm$ 0.1 , extragalactic absorption $N_{H}<1 \times 10^{20} \mathrm{H} \mathrm{cm}^{-2}, \chi_{v}^{2}=1.25$, 116 d.o.f.). The same spectral model results in a spectral index of $\beta=1.2 \pm 0.1$ and $\beta=0.9 \pm 0.2\left(\chi_{v}^{2}=1.30,50\right.$ d.o.f. $)$ during the plateau phase and the following decay respectively. Note that the galactic hydrogen column density along the direction of the GRB is $N_{H}=3.7 \times 10^{21} \mathrm{H} \mathrm{cm}^{-2}$.

\section{Discussion}

\subsection{The giant flare of GRB 060904B}

Giant X-ray flares such as the one observed for GRB 060904B have also been observed for other GRBs (see Burrows et al. 2007; Chincarini et al. 2007, for a review). In particular, the giant flare associated with GRB 050502B was studied in detail by Falcone et al. (2006). Compared with the giant X-ray flare of GRB 060904B, the similarities are striking. GRB 070704 is another example of a GRB with a large X-ray flare $~ 300 \mathrm{~s}$ after the trigger (Godet \& Sakamoto 2007).

First, as for GRB 050502B, we find that the spectrum of GRB 060904B during the flare cannot be fitted by a power law, unless allowing for a variable absorption. The latter shows a significant increase at the onset of the flare, followed by a decrease (which is unusual behavior). We thus modeled the spectra using a Band model and a cut-off model, finding a better explanation. The consistency with the Band model (or cutoff power law) suggests that the flare could be caused by the same mechanism powering the prompt emission, as in a late time internal shock scenario, which was suggested to explain the GRB 050502B giant flare.

The spectral and temporal analysis of the flare show other similarities with the case of GRB 050502B, such as: i) the increasing softening of the spectrum as the flare develops (see Fig. 3); ii) the hard band decaying more quickly than the soft band after the peak of the flare ; iii) the soft band having a shallower decay relative to the slope of the flare rise (see Table 6).

The temporal decay observed before the flare seems to extrapolate to 4000-5000 s after the trigger, with no evident increase in the pre-flare versus the post-flare light curve normalization. This also suggests that the X-ray flare could be produced by an additional mechanism, independent to the one generating the underlying emission. Such emission, in turn, could be interpreted as resulting from the superposition of a decaying prompt and a rising X-ray afterglow component, as proposed by Willingale et al. (2007) to interpret the typical "steep-flat-steep" behavior of Swift XRT light curves. In this scenario, the temporal break observed in the X-ray light curve around $(5 \pm 1) \times 10^{3} \mathrm{~s}$ could mark the transition from the "flat phase" to the "steep" afterglow-dominated phase. We note that the observed steepening in the X-ray light curve $(\Delta \alpha=0.56 \pm$ 0.11 ) is not consistent with a spectral break within a standard afterglow scenario (that would require $\alpha_{1 x}-\alpha_{2 x}=0.25$ ) nor with 


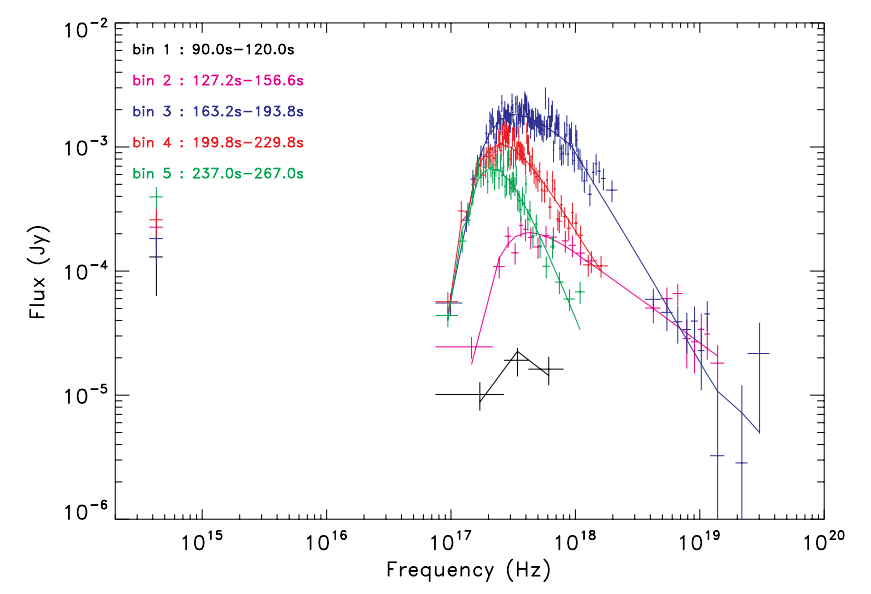

Fig. 7. Evolution of the spectral emission distribution of GRB 060904B according to the first bins corresponding to TAROT exposures. The theoretical model represented is a Band function. It is not possible to reconcile optical and X-ray data with only power-law and extinction. This argues that the optical emission has a different origin to that of the X-ray flare.

an achromatic jet break (the optical light curve seems to extrapolate without breaks from $\sim 3000$ s to late times, see Fig. 3). In addition, the late-time $(t \gtrsim 5000 \mathrm{~s})$ optical-to-X-ray temporal and spectral indices verify (within the errors) the closure relations expected in a standard afterglow scenario, when $v_{m}$ is below the optical band and $v_{c}$ between the optical and the X-ray band (see e.g. Zhang \& Meszaros 2004).

We thus conclude that the giant X-ray flare of GRB 060904B may be due to a late internal shock.

\subsection{Optical rising}

An optical rising has been observed for both GRB 060904B and GRB 070420. For GRB 070420, the rising occured during the X-ray steep decay (see Fig. 4). $V$-band UVOT observations (Immler et al. 2007) suggest an achromatic behavior.

Differently from the cases of GRB 050502B or GRB 070704, which had no optical counterpart, for GRB 060904B an optical emission is observed: the optical light curve rises smoothly during the whole X-ray flare, and shows a maximum after the end of the X-ray flare. One could consider the possibility that this flare is correlated with the optical rise. In fact, the broad band data suggest a temporal sequence, with a flare moving from the $\gamma$-ray band to the optical band (see Fig. 3). In such a case the X-ray and optical emissions are produced by the same mechanism (internal shock, see Sect. 4.1), with the peak of the emission moving from the BAT to optical bands. Hence, near the peak of the emission one should expect the same spectro-temporal behavior. However, the observed X-ray rise and decay temporal slopes are significantly steeper than the optical ones (see Table 6). This suggests that the optical rising and the BAT-XRT flare are not correlated. We also note that extrapolating in the optical band the best fit Band models listed in Table 5, results in an overestimation of the optical flux. We can reconcile the observed optical data with the high energy extrapolation only by invoking a strong extinction. Moreover, such extinction should increase as the flare develops, which is an unsual behavior.

A similar rise was observed in the near infrared light curves of GRB 060418 and GRB 060607A (Molinari et al. 2007), together with a simultaneous X-ray emission characterized by the
Table 6. Summary of temporal indixes for the optical and X-ray light curves of GRB 060904B. We report a decay with positive index and an increase with negative index.

\begin{tabular}{ccc}
\hline \hline Part & Optical index & X-ray index \\
\hline Pre X-flare & n.d. & $0.86 \pm 0.05$ \\
X-flare rise & $-0.82 \pm 0.15$ & $-17.83 \pm 0.02$ \\
X-flare decay & $-0.82 \pm 0.15$ & $6.1 \pm 0.4$ \\
Optical Peak & 0 & $0.86 \pm 0.05$ \\
First optical decay & $1.00 \pm 0.18$ & $0.86 \pm 0.05$ \\
Optical plateau & 0 & $0.86 \pm 0.05$ \\
Final decay before X-break & $1.03 \pm 0.03$ & $0.86 \pm 0.05$ \\
Final decay after X-break & $1.03 \pm 0.03$ & $1.42 \pm 0.10$ \\
\hline
\end{tabular}

presence of various flares. In these cases, the low-energy optical rise was interpreted as the peak of the afterglow emission which, in the standard thin shell case, is predicted to occur around the deceleration time $t_{\mathrm{dec}} \sim t_{\text {peak }}$ (Sari \& Piran 1999; Kobayashi \& Zhang 2007). Up to now, only a tenth of optical afterglows have been observed sufficiently early, and time-sampled sufficiently well for the optical rising to be clearly identified. Theoretically, if the rise has to be ascribed to the beginning of the afterglow, in a standard ISM scenario the temporal index $\alpha_{1}$ is expected to be $\leq-2$ (Sari \& Piran 1999), much steeper than our observed value. In the case of a fireball expanding in a wind environment, the rise could be less steep and also followed by a plateau (see e.g. Fig. 1 of Wu et al. 2003). In such a case, we would expect the late optical light curve to decrease with the same temporal index, or more steeply, than in the X-rays. However, as discussed in Sect. 4.1, if we interpret the X-ray emission at $\sim 5000 \mathrm{~s}$ as the link to the "standard" afterglow phase, then the hypothesis of an expansion in a wind environment does not agree with the late time observations. In fact, at $t \gtrsim 5000 \mathrm{~s}$, the X-ray decay is steeper than the optical one.

Other phenomena can also contribute to a late rising of the optical afterglow: i) an off axis line of sight (Granot et al. 2002) as was supposed in the case of GRB 060206 by Wozniak et al. (2006) for which the fluence was very low $(8.4 \pm 0.4 \times$ $10^{-7} \mathrm{erg} \mathrm{cm}^{-2}$ in the $15-150 \mathrm{keV}$ range). This is not the case for GRB 070420 for which the fluence is exceptionally high. Moreover, in the case of an off-axis observer, the X-ray and the optical light curves must follow the same behavior, which is not the case of the two studied GRBs. ii) The circumstellar medium could be very dense and the extinction very high at the beginning of the optical afterglow, and could decrease later because of the dust destruction implied by the burst blast wave (Perna \& Loeb 1998). This is probably not the case of GRB 060904B because optical emission was detected by ROTSE during the prompt emission and color indexes do not show a red excess near $t_{\text {peak }}$. Optical indexes prove that the extinction was not high in both GRBs. iii) In the case of a smooth gradient of interstellar material density (Tam et al. 2005), the rise is explained by an appropriate density profile if the optical emission is below the cooling frequency (Sari et al. 1998). iv) Reverse shock emission could be invoked. In this scenario, one could interpret the optical bump of GRB 060904B as an optical flash, and the following plateau as the result of the forward shock emission adding up to the reverse shock one: see Fig. 2 of Sari \& Piran (1999). However, in such a case, the extrapolated power-law behavior of both the rise and decay of the reverse shock optical emission are expected to be somewhat steeper than those observed in our case (Sari \& Piran 1999; Kobayashi \& Zhang 2007). v) the optical rise and decay temporal indexes of $\alpha_{1}=-0.82 \pm 0.15$ and 
$\alpha_{2}=1.00 \pm 0.18$, could be marginally accommodated within a standard afterglow scenario, by having the $v_{m}$ cross the optical band.

\subsection{Optical plateau}

Some GRBs exhibit an optical plateau that consists of a phase of very shallow decay starting a few minutes after the trigger (typically $5 \mathrm{~min}$ in the burst rest frame) and lasting a few more minutes. The plateau can appear simultaneously in the optical and in the X-ray band (e.g. GRB 050801 De Pasquale et al. 2007); in some cases we observe an early re-brightening rather than a plateau; in other events a plateau appears in the optical on longer timescales, e.g. GRB 060206 (Stanek et al. 2007; Monfardini et al. 2006) with no correlation with any X-ray flattening.

Of the two GRB analyzed in this work, only GRB 060904B shows evidence of an optical plateau. Several scenarios could explain such a plateau. In the standard fireball scenario a nearly flat optical light curve (temporal decay index $\alpha=-0.25$ ) can be produced by the forward shock when the fireball is in the fast cooling regime with the observational frequency between the cooling frequency $v_{c}$ and the injection frequency $v_{m}$, see Fig. 2 of Sari \& Piran (1999). For GRB 060904B, this ordering of characteristic emission frequencies cannot be reconciled with the late ( $t \gtrsim 5000 \mathrm{~s}$ ) optical-to-X-ray data. Another possibility is that the plateau is produced by a patchy jet, i.e. by a collimated fireball characterized by a non-uniform distribution of energy (Kumar $\&$ Piran 2000). In such a case we expect a large bump followed by a plateau (see Fig. 6 of Zhang et al. 2006), as we observe in GRB 060904B. However, we should then see the same behavior roughly simultaneously in all bands, which is inconsistent with GRB 060904B X-ray data. A last hypothesis for the origin of the optical plateau is that it is produced by a late energy injection in the fireball. Late energy injection can be ascribed to a long lasting central engine activity, or to a refreshed shock associated with a short living central engine that is releasing its energy with a variety of Lorentz factors (Rees \& Meszaros 1998). This hypothesis was also proposed by De Pasquale et al. (2007) to explain the plateau observed simultaneously in optical and X-ray in GRB 050801. In our case, it is difficult to conclude about the presence of a simultaneous X-ray plateau, also because of the possible contamination by internal shock emission.

We observe a large optical plateau when a very large X-ray flare is observed (GRB 060904B) and no plateau when there is no X-flare (GRB 070420). There are not enough observations from other GRBs to confirm this point but this correlation must be addressed by acquiring data of early afterglows in both X-ray and optical wavelengths.

\section{Conclusion}

The optical emissions of GRB 060904B and GRB 070420 are found to increase from the end of the prompt phase, reaching a maximum of brightness at $t_{\text {peak }}=9.2 \mathrm{~min}$. and $3.3 \mathrm{~min}$. respectively and then decreasing. GRB 060904B presents a large optical plateau and a huge X-ray flare. We argue that the huge $\mathrm{X}$-flare occurring near $t_{\text {peak }}$ is produced by an extended internal engine activity. Its presence during the optical rise is only a coincidence, and is not related to the optical flare. GRB 070420 observations would support this fact because there was no X-flare during the optical peak. We have proposed that the nature of the optical plateau of GRB 060904B, while not completely elucidated, could be related to late energy injection.

Acknowledgements. B. Gendre acknowledges support from COFIN grant 2005025417. The TAROT telescope has been funded by the Centre National de la Recherche Scientifique (CNRS), Institut National des Sciences de l'Univers (INSU) and the Carlsberg Fundation. It has been built with the support of the Division Technique of INSU. We thank the technical staff contributing to the TAROT project: G. Buchholtz, J. Eysseric, M. Merzougui, C. Pollas, and P. \& Y. Richaud. We thank E. Rykoff who provides some images of ROTSE-III and A. Kann who suggested some useful corrections. S. Cutini, B. Preger \& G. Stratta acknowledge support of ASI contract 1/024/05/0.

\section{References}

Akerlof, C., Balsano, R., Barthelmy, S., Bloch, J., et al. 1999, Nature, 398, 400 Berger, E., Kulkarni, S. R., Fox, D. B., et al. 2005, ApJ, 634, 501

Boër, M., Atteia, J. L., Damerdji, Y., et al. 2006, ApJ, 638, L71

Bringer, M., Boër, M., Peignot, C., et al. 2001, Exper. Astrophys, 12, 34

Burrows, D. N., Falcone, A., Chincarini, G., et al. 2007, Philos. Trans. A, accepted [arXiv: astro-ph/0701046]

Chincarini, G., Moretti, A., Romano, P., et al. 2007, ApJ, submitted [arXiv: astro-ph/0702371]

Cobb, B. E., \& Bailyn, C. D. 2007, GCNC, 5525

Costa, E., Frontera, F., Heise, J., et al. 1997, Nature, 387, 783

D’Avanzo, P., Antonelli, L. A., Calzoletti, L., et al. 2007, GCNC, 6331

de Pasquale, M., Piro, L., Gendre, B. 2006, A\&A, 455, 813

de Pasquale, M., Oates, S. R., Page, M. J., et al. 2007, MNRAS, submitted [arXiv:astro-ph/0703447]

Fox, D. 2005, in Proc. Gamma Ray Bursts in the Swift Era, ed. S. Holt, N. Geherels, \& J. Nousek, AIPC

Gehrels, N., Chincarini, G., Giommi, P., et al. 2004, ApJ, 611, 1005

Gendre, B., Corsi, A., \& Piro, L. 2006, A\&A, 455, 803

Godet, O., \& Sakamoto, T. 2007, GCNC, 6598

Granot, J., Panaitescu, A., Kumar, P., et al. 2002, ApJ, 570, L61

Grupe, D., Barthelmy, S. D., Chester, M. M., et al. 2006, GCNC, 5505

Falcone, A. D., Burrows, D. N., Lazzati, D., et al. 2006, ApJ, 641, 1010

Fugazza, D., et al. 2006, GCNC, 5513

Immler, S., Marshall, F. E., Holland, S. T., et al. 2007, GCNC, 6336

Kann, A., Klose, S. Zhang, B., et al. 2008, ApJ, submitted, [arXiv: 0712 . 2186]

Klotz, A., Boër, M., \& Atteia, J. L. 2005, GCNC, 3473

Klotz, A., Boer, M., Atteia, J. L., et al. 2006a, GCNC, 5508

Klotz, A., Gendre, B., Stratta, G., et al. 2006b, A\&A, 451, L39

Kobayashi, S., \& Zhang, B. 2007, ApJ, 655, 973

Kumar, P., \& Piran, T. 2000, ApJ, 532, 286

Markwardt, C., Barbier, L., Barthelmy, S. D., et al. 2006, GCNC, 5520

Molinari, E., Vergani, S. D., Malesani, D., et al. 2007, A\&A, 469, L13

Monfardini, A., Kobayashi, S., Guidorzi, C., et al. 2006, ApJ, 648, 1125

O’Brien, P. T., Willingale, R., \& Osborne, J. 2006, ApJ, 647, 1213

Pélangeon, A. \& Atteia, J.-L. 2007, GCNC, 6345

Perna, R., \& Loeb, A. 1998, ApJ, 501, 467

Rees, M., \& Meszaros, P. 1998, ApJ, 496, L1

Romano, P., Campana, S., \& Chincarini, G. 2006, A\&A, 456, 917

Roming, P., Schlady, P., Fox, D., et al. 2006, ApJ, 652, 1416

Rykoff, E. S., Yost, S. A., Krimm, H. A., et al. 2005, ApJ, 631, L121

Rykoff, E. S., Rykoff, E. S., Rujopakarn, W., Yuan, F. et al., 2006, GCNC, 5504

Sari, R., \& Piran, T. 1999, ApJ, 520, 641

Sari, R., Piran, T., \& Narayan, R. 1998, ApJ, 497, L17

Schlegel, D. J., Finkbeiner, D. P., \& Davis, M. 1998, ApJ, 500, 525

Simon, V., Hudec, R., Pizzichini, G., et al. 2001, A\&A, 366, 110

Stamatikos, M., Burrows, D. N., Cusumano, G., et al. 2007a, GCNC, 6330

Stamatikos, M., et al. 2007b, GCN-Report-48.1

Stanek, K. Z., Dai, X., Prieto, J. L., et al. 2006, ApJ, 654, L21

Stratta, G., Perri, M., Burrows, D. N., et al. 2007, GCNC, 6337

Tam, P. H., Pun, C. S. J., Huang, Y. F., et al. 2005, New Astron., 10, 535

Vestrand, W. T, Wozniak, P. R., Wren, J. A., et al. 2006, Nature, 442, 172 Wijers, R. A., \& Galama, T. J. 1999, ApJ, 523, 177

Willingale, R., O’Brien, P. T., Osborne, J. P., et al. 2007, ApJ, 662, 1093

Wozniak, P. R., Vestrand, W. T., Wren, J. A., et al. 2006, ApJ, 642, L99

Wu, X. F., Dai, Z. G., Huang, Y. F., et al. 2003, MNRAS, 342, 1131

Zhang, B., \& Meszaros, P. 2004, Int. J. Mod. Phys., A19, 2385

Zhang, B., Kobayashi, S., \& Meszaros, P. 2003, ApJ, 595, 950

Zhang, B., Fan, Y. Z., \& Dyks, J. 2006, ApJ, 642, 354 